\title{
Widespread and evolutionary analysis of a MITE family Monkey King in Brassicaceae
}

Shutao Dai ${ }^{1}$, Jinna Hou ${ }^{1,2}$, Yan Long ${ }^{1}$, Jing Wang ${ }^{1}$, Cong Li ${ }^{1}$, Qinqin Xiao ${ }^{1}$, Xiaoxue Jiang ${ }^{1}$, Xiaoxiao Zou', Jun Zou ${ }^{1}$ and Jinling Meng ${ }^{1 *}$

\begin{abstract}
Background: Miniature inverted repeat transposable elements (MITEs) are important components of eukaryotic genomes, with hundreds of families and many copies, which may play important roles in gene regulation and genome evolution. However, few studies have investigated the molecular mechanisms involved. In our previous study, a Tourist-like MITE, Monkey King, was identified from the promoter region of a flowering time gene, BnFLC.A10, in Brassica napus. Based on this MITE, the characteristics and potential roles on gene regulation of the MITE family were analyzed in Brassicaceae.

Results: The characteristics of the Tourist-like MITE family Monkey King in Brassicaceae, including its distribution, copies and insertion sites in the genomes of major Brassicaceae species were analyzed in this study. Monkey King was actively amplified in Brassica after divergence from Arabidopsis, which was indicated by the prompt increase in copy number and by phylogenetic analysis. The genomic variations caused by Monkey King insertions, both intra- and inter-species in Brassica, were traced by PCR amplification. Genomic sequence analysis showed that most complete Monkey King elements are located in gene-rich regions, less than $3 \mathrm{~kb}$ from genes, in both the B. rapa and A. thaliana genomes. Sixty-seven Brassica expressed sequence tags carrying Monkey King fragments were also identified from the NCBI database. Bisulfite sequencing identified specific DNA methylation of cytosine residues in the Monkey King sequence. A fragment containing putative TATA-box motifs in the MITE sequence could bind with nuclear protein(s) extracted from leaves of B. napus plants. A Monkey King-related microRNA, bna-miR6031, was identified in the microRNA database. In transgenic A. thaliana, when the Monkey King element was inserted upstream of $35 \mathrm{~S}$ promoter, the promoter activity was weakened.

Conclusion: Monkey King, a Brassicaceae Tourist-like MITE family, has amplified relatively recently and has induced intra- and inter-species genomic variations in Brassica. Monkey King elements are most abundant in the vicinity of genes and may have a substantial effect on genome-wide gene regulation in Brassicaceae. Monkey King insertions potentially regulate gene expression and genome evolution through epigenetic modification and new regulatory motif production.
\end{abstract}

Keywords: Brassicaceae, Brassica, Miniature inverted repeat transposable elements, Monkey King, Tourist-like MITE, DNA methylation, bna-miR6031

\section{Background}

Miniature inverted repeat transposable elements (MITEs) are a class of non-autonomous DNA transposable elements (classII) [1]. They were first described in the mutated maize allele $w x-B 2[2]$ and subsequent studies have revealed that MITEs are predominant in almost all

\footnotetext{
* Correspondence: jmeng@mail.hzau.edu.cn

${ }^{1}$ National Key Lab of Crop Genetic Improvement, Huazhong Agricultural University, Wuhan, Hubei 430070, China

Full list of author information is available at the end of the article
}

plants and animals. They often have terminal inverted repeats (TIRs) and target site duplications (TSDs) at the ends of the elements. Based on TSD sequences, earlier studies showed that MITEs were mainly classified into two super-families: Tourist-like MITEs (3-bp, TAA) [2, 3] and Stowaway-like MITEs (2-bp, TA) [4]. Studies have shown that MITEs may originate from internal deletion of corresponding autonomous transposable elements; thus, Tourist and Stowaway MITE super-families are assumed originated from PIF/Harbinger and Tc1/mariner elements, 
respectively [5-7]. Later studies indicated that some MITEs were derived from other autonomous DNA transospons, such as hAT transposons [8, 9] and Mutator transposons [10]. In addition, due to ambiguous TSD and/ or TIR features, Some MITEs were annotated as unknown super-families [11].

There are hundreds of families of MITEs and they are present in high copy numbers, making them important genome constituents. These elements are widely, but not randomly, distributed in the genome, and their distribution density in each chromosome varies [12]. Thousands of MITE copies provide potential resources for genomic structure variation and may fuel genomic evolution. Recent activities of MITEs have produced abundant MITE-derived polymorphisms, which may contribute to considerable phenotypic diversity in rice [13]. mPing, a Tourist-like MITE, which originated from an internal deletion of a transposase-encoding element Ping, is activated by tissue culture and $\gamma$-ray irradiation in rice[14-16]. mPing insertions presented different profiles (from 50 to 1,000 copies) among four rice strains under selection during domestication [17]. It was suggested that some new alleles induced by mPing insertions might benefit the host by creating potentially useful allelic variants and novel, stressinducible regulatory networks $[17,18]$.

Although selection pressure tends to eliminate most insertions that reside in gene exons and introns in the early stage of MITE amplification in the genome [17], studies have still found that more ancient MITE subfamilies are preferentially associated with genes [19]. This suggested that MITEs may be associated with the expression of neighboring genes. Much recent research has focused on the function of MITEs in gene regulation. kiddo, a MITE located in the rice ubiquitin2 promoter, has a dual function in gene regulation: its presence not only increases transcription rates but induces epigenetic modifications [20]. Small RNAs regulate the activity of transposable elements via a class of transposable element (TE)-derived 24-nt siRNAs [21]. In Solanaceae, MITEs generate small RNAs that are mostly $24 \mathrm{nt}$ in length and MITE siRNA biogenesis involves DICER-LIKE 3, RNAdependent RNA polymerase 2 , and possibly DICER-LIKE 4 [22].

Brassica, a close relative of Arabidopsis, is an agriculturally important genus that includes a wide range of diploid and allotetraploid species, including oil crops, vegetables, and forages. $B$. napus, an allotetraploid species (AACC, $2 n=2 x=38$ ), originated from natural hybridization between the ancestral forms of the diploid species $B$. rapa (AA, $2 n=2 x=20$ ) and B. oleracea (CC, $2 n=2 x=18) \sim 7500$ years ago $[23,24]$. The Brassica A and $C$ genomes were estimated to have diverged $\sim 4.6$ million years ago [25]. The same sets of genomes in $B$. napus and its progenitors were defined as subgenomes of each other. The A genomes in B. rapa and B. napus were assigned the $A^{\mathrm{r}}$ and $\mathrm{A}^{\mathrm{n}}$ subgenomes, respectively; $\mathrm{C}^{\mathrm{o}}$ and $\mathrm{C}^{\mathrm{n}}$ represent the $\mathrm{C}$ genome in $B$. oleracea and $B$. napus, respectively [26,27]. Sequence-level comparative analysis has revealed that the similarity between the $\mathrm{A}^{\mathrm{r}}$ and $\mathrm{A}^{\mathrm{n}}$ subgenomes is $97.5 \pm 3.1 \%$, and is $93.1 \pm 4.9 \%$ between the $A^{r}$ and $C^{n}$ subgenomes [28]. It has been suggested that transposable elements contribute to sequence variation in the $A$ and $C$ genomes [23, 28, 29].

A Stowaway-like MITE, BraSto, first reported in $B$. rapa, was found in the gene space and is still active both in diploid and allotetraploid Brassica species [30]. In B. napus, a Tourist-like MITE, Monkey King, was identified in the promoter region of BnFLC.A10, a homologue of Arabidopsis FLOWERING LOCUS C $(F L C)$ [31]. In this study, we found that Monkey King elements are not restricted to Brassica species, but are specific to the Brassicaceae family. We further investigated its sequence features, distribution, and phylogenetic relationships, and inferred its potential role in the evolution of Brassicaceae genomes. Monkey King-related intraand inter-species polymorphisms were confirmed experimentally. DNA methylation analysis, electrophoretic mobility shift assay (EMSA) analysis, identification of a Monkey King-related microRNA (miRNA), and transgenic analysis revealed its effects on gene expression and genome evolution in Brassicaceae.

\section{Results}

\section{Characteristics of a Tourist MITE family, Monkey King, in Brassicaceae}

The Monkey King sequence in the promoter of BnFLC.A10 included $14 \mathrm{bp}$ TIRs and was flanked with a trinucleotide TAA TSD, which are typical features of Tourist MITEs (Fig. 1a). An AT-rich core with a 270-bp A/T continuous fragment was found in the internal region of the sequence. A stem-loop formed in the secondary structure, with the TIRs complementing each other (Fig. 1b). Part of the nucleotide sequence seems to translate into amino acid residues, but no complete protein is encoded (data not shown).

From the B. rapa and A. lyrata genome sequences, a total of 1186 and 278 homologous sequences (including complete and partial Monkey King sequences), respectively, were screened in the published plant MITE database (P-MITE) [11]. Although no similar sequence was found in the MITE database of A. thaliana, 52 Monkey King homologous sequences were identified in the $A$. thaliana genome sequence by BLAST analysis (Table 1 ). Monkey King seems to be specific to the Brassicaceae family, because no similar sequences were found in other plant families. Monkey King density analysis of the three published genome sequences showed that the $B$. rapa genome, which is the largest genome in size, has 

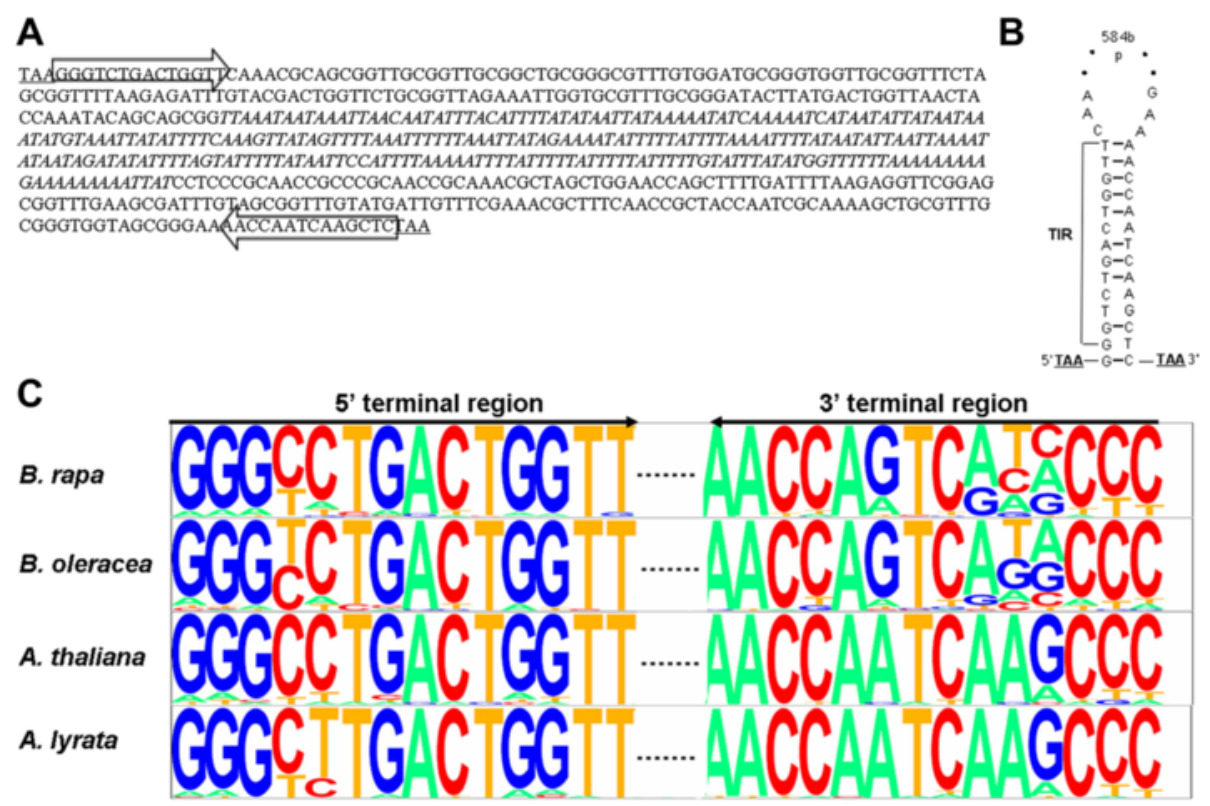

Fig. 1 Identification and classification of Monkey King. (a) Sequence and structural characteristics of the Monkey King insertion in the BnFLC.A10 promoter. The 3-bp TSDs and TIRs are highlighted underlined and framed with arrows at the ends of the sequence, respectively; italics indicate a $270 \mathrm{bp} \mathrm{A} / \mathrm{T}$ continuous fragment in the core region. (b) A Stem-loop structure generated by a pair of 14-bp TIR of the Monkey King insertion. Ten of the 14 nucleotides in each of the TIRs are complementary to each other and the other four nucleotides have mismatches. TSDs are underlined. Dots represent the internal sequence in the Monkey King insertion. (c) Pictogram of TIR sequences obtained from complete Monkey King sequences in B. rapa, B. oleracea, A. thaliana, and A. lyrata. The height of each letter is proportional to the relative frequency of each nucleotide at that position

the highest density (4.18 MITEs/Mb), while the smallest genome (A. thaliana) has the lowest density ( 0.43 MITEs/Mb). In the same species, no significant differences were found in Monkey King density among different chromosomes, except for chromosome 3 from $A$. thaliana and A. lyrata. In silico mapping of 504 complete elements on the $B$. rapa chromosomes also showed that they were approximately evenly distributed in their respective chromosomes (Fig. 2). The physical positions of 504 complete Monkey King elements from the B. rapa genome are listed in Additional file 1. The average length of the complete Monkey King sequences varies significantly among the three genome sequences: the shortest was identified in $B$. rapa, followed by $A$. lyrata, while the longest was from $A$.

Table 1 Distribution of Monkey King elements in B. rapa, A. lyrata and A. thaliana genome

\begin{tabular}{|c|c|c|c|c|c|c|c|c|c|c|c|}
\hline \multicolumn{4}{|l|}{ B. rapa } & \multicolumn{4}{|l|}{ A. lyrata } & \multicolumn{4}{|c|}{ A. thaliana } \\
\hline Chr. no. & $\begin{array}{l}\text { Size of } \\
\text { Chr. (Mb) }\end{array}$ & $\begin{array}{l}\text { No. of } \\
\text { elements }\end{array}$ & $\begin{array}{l}\text { MITE } \\
\text { density }^{\text {a }}\end{array}$ & Chr. no. & $\begin{array}{l}\text { Size of } \\
\text { Chr. (Mb) }\end{array}$ & $\begin{array}{l}\text { No. of } \\
\text { elements }\end{array}$ & $\begin{array}{l}\text { MITE } \\
\text { density }\end{array}$ & Chr. no. & $\begin{array}{l}\text { Size of } \\
\text { Chr. (Mb) }\end{array}$ & $\begin{array}{l}\text { No. of } \\
\text { elements }\end{array}$ & $\begin{array}{l}\text { MITE } \\
\text { density }\end{array}$ \\
\hline$\overline{A 01}$ & 28.61 & 134 & 4.68 & Chr.1 & 33.13 & 52 & 1.57 & Chr.1 & 30.43 & 12 & 0.39 \\
\hline $\mathrm{A} 02$ & 27.85 & 131 & 4.70 & Chr.2 & 19.32 & 28 & 1.45 & Chr.2 & 19.70 & 12 & 0.61 \\
\hline A03 & 31.72 & 139 & 4.38 & Chr.3 & 24.46 & 18 & 0.74 & Chr.3 & 23.46 & 5 & 0.21 \\
\hline A04 & 18.97 & 101 & 5.32 & Chr.4 & 23.33 & 25 & 1.07 & Chr.4 & 18.59 & 9 & 0.48 \\
\hline A05 & 23.94 & 110 & 4.59 & Chr.5 & 21.22 & 34 & 1.60 & Chr.5 & 26.98 & 14 & 0.52 \\
\hline A06 & 26.27 & 113 & 4.30 & Chr.6 & 25.11 & 27 & 1.08 & & & & \\
\hline $\mathrm{A} 07$ & 22.59 & 119 & 5.27 & Chr.7 & 24.65 & 45 & 1.83 & & & & \\
\hline A08 & 21.60 & 94 & 4.35 & Chr.8 & 22.95 & 31 & 1.35 & & & & \\
\hline A09 & 37.12 & 184 & 4.96 & Uncertain & 12.59 & 18 & 1.43 & & & & \\
\hline $\mathrm{A} 10$ & 17.60 & 62 & 3.52 & & & & & & & & \\
\hline Uncertain & 27.58 & 99 & 3.59 & & & & & & & & \\
\hline Total & 283.84 & 1186 & 4.18 & Total & 206.67 & 278 & 1.35 & Total & 119.67 & 52 & 0.43 \\
\hline
\end{tabular}

${ }^{\mathrm{a} N o . ~ o f ~ M I T E s ~ p e r ~ M b ~}$ 


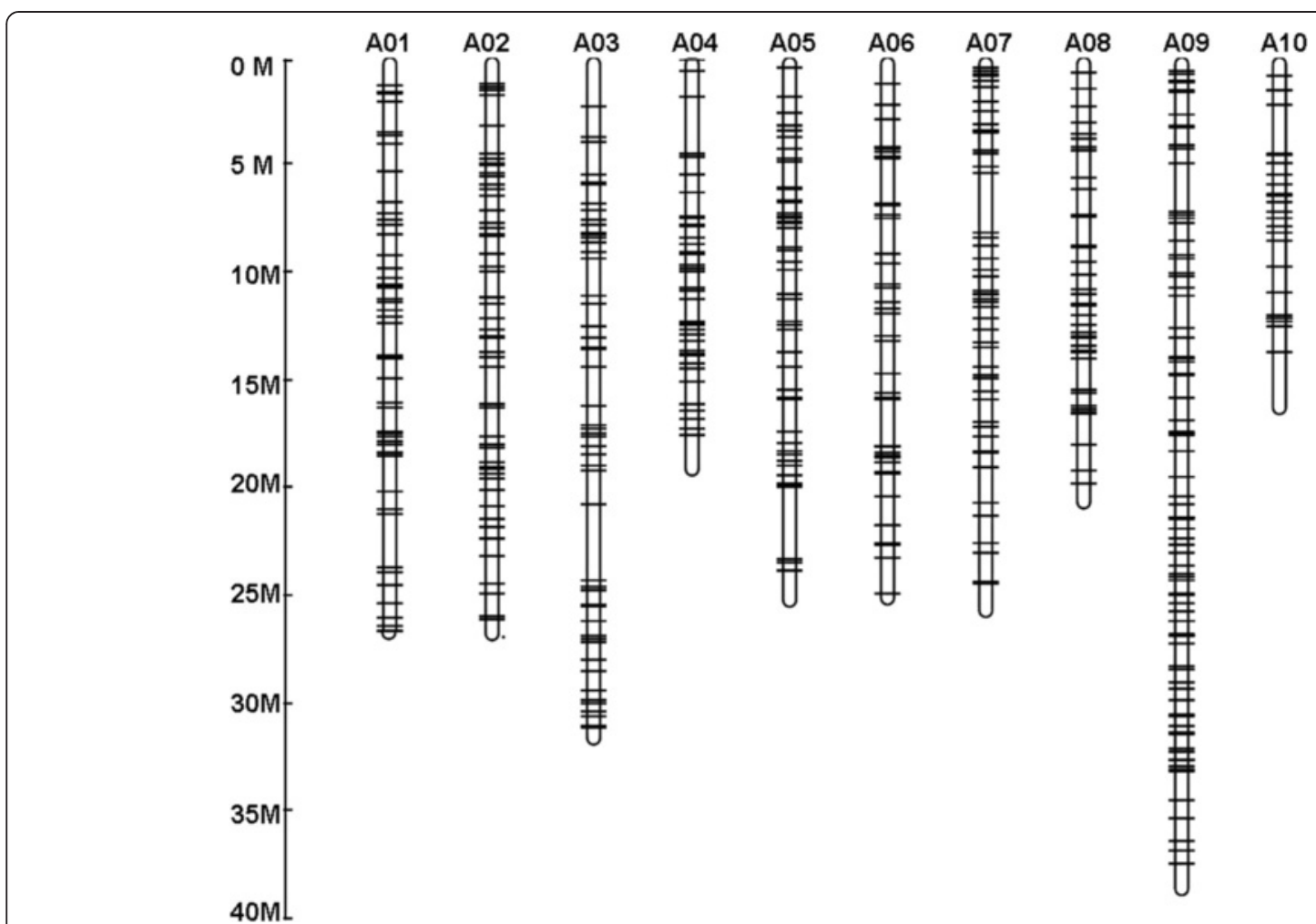

Fig. 2 In silico mapping of 504 complete Monkey King elements in the genome of B. rapa. The physical positions details for the Monkey King elements are listed in Additional file 1

thaliana. The average AT-contents of these sequences vary slightly among the three species (Table 2). However, different Monkey King sequences have considerable variation in nucleotide composition in the same genome, especially in the B. rapa genome (the AT-content ranged from 50.7 to $79.4 \%)$. Correlation analysis between the AT-content and the length of complete Monkey King sequences showed that longer Monkey King sequences have relatively higher AT-contents in the $B$. rapa genome ( $r=0.7, P<0.01)$ (Fig. 3 ).

Monkey King TIR consensus sequences were identified in four Brassicaceae genomes: B. rapa, B. oleracea, A. thaliana and A. lyrata. For B. oleracea, 70 complete Monkey King sequences were identified in the preliminary assembled $B$. oleracea genome sequence using BLAST analysis. The TIR sequences are strongly conserved among these
Brassicaceae genomes (Fig. 1c). In general, one specific base occupied the highest proportion for one position. It seems that TIR sequences from the two Brassica genomes are more variable than those of the two Arabidopsis genomes, especially at the $4^{\text {th }}$ and $5^{\text {th }}$ nucleotides in the $3^{\prime}$ terminal regions. Additionally, there was a distinct difference $(A \rightarrow G$ transition) at the $9^{\text {th }}$ nucleotide in the $3^{\prime}$ terminal regions between the Brassica and Arabidopsis genomes.

\section{Phylogenetic analysis of the Monkey King elements in four Brassicaceae genomes}

All the complete Monkey King sequences mined from the four Brassicaceae genomes were used for phylogenetic analysis. In addition, the Monkey King sequence in the promoter of BnFLC.A10 from B. napus was included. From the phylogenetic tree (Fig. 4), the Monkey

Table 2 Nucleotide composition of complete Monkey King sequences in B. rapa, A. lyrata and A. thaliana genomes

\begin{tabular}{|c|c|c|c|c|c|c|c|}
\hline \multirow[t]{2}{*}{ Species } & \multirow{2}{*}{$\begin{array}{l}\text { No. of } \\
\text { complete } \\
\text { sequences }\end{array}$} & \multicolumn{3}{|c|}{ The length of complete sequences } & \multicolumn{3}{|c|}{ The AT-content of complete sequences } \\
\hline & & Min. & Max. & Average & Min. & Max. & Average \\
\hline B. rapa & 504 & $322 \mathrm{bp}$ & $791 \mathrm{bp}$ & $545 \pm 94 \mathrm{bp}$ & $50.70 \%$ & $79.40 \%$ & $67.0 \pm 5.0 \%$ \\
\hline A. lyrata & 55 & $452 \mathrm{bp}$ & 796 bp & $619 \pm 46 \mathrm{bp}$ & $61.2 \%$ & $72.5 \%$ & $65.7 \pm 2.3 \%$ \\
\hline A. thaliana & 38 & $590 \mathrm{bp}$ & $1158 \mathrm{bp}$ & $890 \pm 150 \mathrm{bp}$ & $65.3 \%$ & $71.9 \%$ & $67.0 \pm 1.4 \%$ \\
\hline
\end{tabular}




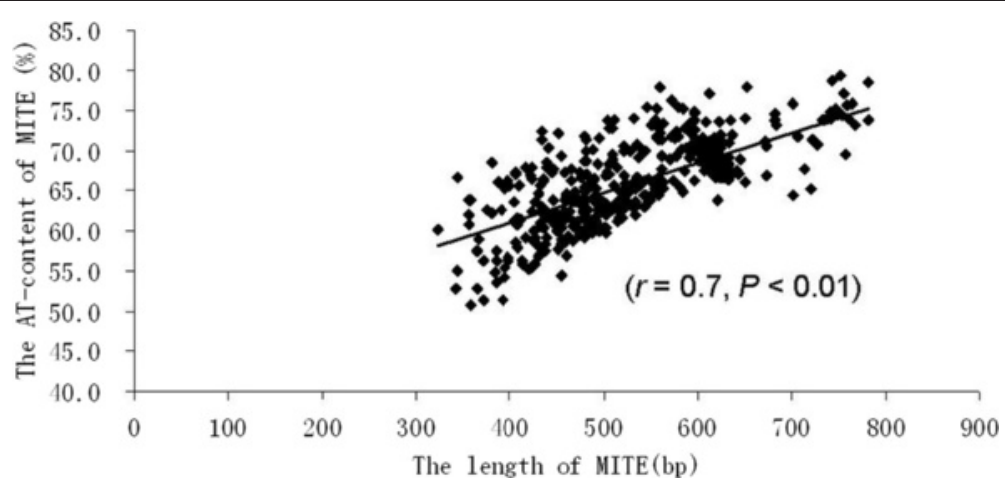

Fig. 3 The correlation between the AT-content and the length of complete Monkey King sequences in B. rapa genome

King members of $A$. thaliana and A. lyrata could be distinguished clearly from the Brassica members. By contrast, the Monkey King members from the two Brassica genomes were interspersed with each other and could not be well separated, which indicated that they have high sequence similarity. However, some members from the same Brassica genome formed a small cluster, indicating that they had been rapidly amplified in their respective genomes after $\mathrm{A}$ - and $\mathrm{C}$ - genomic species differentiation. The Monkey King member in the $B n F L C . A 10$ promoter clustered into an A genome specific group, which indicated that the insertion may be from A genome in B. napus. In addition, different small clusters contained Monkey King members from $B$. rapa and $B$. oleracea, which indicated that they might have diverged before the differentiation of the A and $\mathrm{C}$ genomes.

\section{The preferred insertion sites of Monkey King elements}

The insertion sites of the 504 and 38 complete Monkey King elements were inspected in the $B$. rapa and $A$. thaliana genomes using the annotated genome databases, respectively. In the $B$. rapa genome, $74.4 \%$ of the elements were inserted in gene-rich regions, less than $3 \mathrm{~kb}$ from genes. Among them, nearly half of the members were within less than $1 \mathrm{~kb}$ from a gene, and a few members $(24,4.8 \%)$ were located within introns of genes (Table 3 ). In the $A$. thaliana genome, notably, 92.1 $\%$ of the elements were located in the gene-rich regions, while only three members $(7.9 \%)$ were more than $3 \mathrm{~kb}$ from a gene. Most of the members (26/38) were within less than $1 \mathrm{~kb}$ from a gene, and two $(5.1 \%)$ were within introns (Table 3). We also calculated the distance between the Monkey King elements and untranslated regions (UTR) of genes in A. thaliana (Additional file 2). $47.3 \%$ of the members (18/38) were within less than $0.5 \mathrm{~kb}$ from a UTR. Moreover, two members fell within UTR regions. The details of the insertion sites of these complete Monkey King elements from the two species are listed in Additional files 1 and 2. Although the Monkey
King copy ratios in different genomic regions showed some differences between $B$. rapa and $A$. thaliana, a similar trend was observed in the genomic locations between the two species: the closer to a gene, the higher the ratio of Monkey King insertions. To further investigate the relationship between Monkey King and genes, we examined potential transcriptional activity of Monkey King by searching the Brassica expressed sequence tag (EST) database at NCBI. Sixty-seven ESTs carrying Monkey King fragments were mined from $B$. rapa, B. oleracea and $B$. napus. Thirty ESTs matched with annotated $B$. rapa and (or) A. thaliana genes (Additional file 3). According to the corresponding gene structure, the Monkey King fragments from these ESTs were mainly located in 3'UTR and intron regions. Although more Monkey King elements were inserted in the $5^{\prime}$ flanking sequences relative to the 3 ' flanking sequences of genes, only one Monkey King fragment from a EST was found in a 5'UTR of a gene.

Intra- and inter-species polymorphisms caused by Monkey King insertions in Brassica species

To confirm if the Monkey King insertions were actually species-specific or cause intra- and inter-species polymorphisms in Brassica species, PCR amplification was carried out using primers designed against the Monkey King flanking sequences. Sequence comparisons further corroborated the PCR results (Fig. 5). Two Monkey King members, SQ045001123 and SQ045005824, were only detected in B. rapa and not in B. napus or B. oleracea (Fig. 5a and b); the Monkey King member C01-1 was only observed in B. oleracea and not B. napus or B. rapa (Fig. 5c). Those insertions are probably species-specific and were resulted from independent activation after speciation. The Monkey King member SQ045004581 was detected in both $B$. rapa and B. napus, but not in $B$. oleracea (Fig. 5d), while the Monkey King member C016 was detected in both B. oleracea and B. napus, but not in $B$. rapa (Fig. 5e). We deduced that the two members are $\mathrm{A} / \mathrm{C}$ genome-specific and were inserted into the Brassica genome after B. rapa and B. oleracea separated 


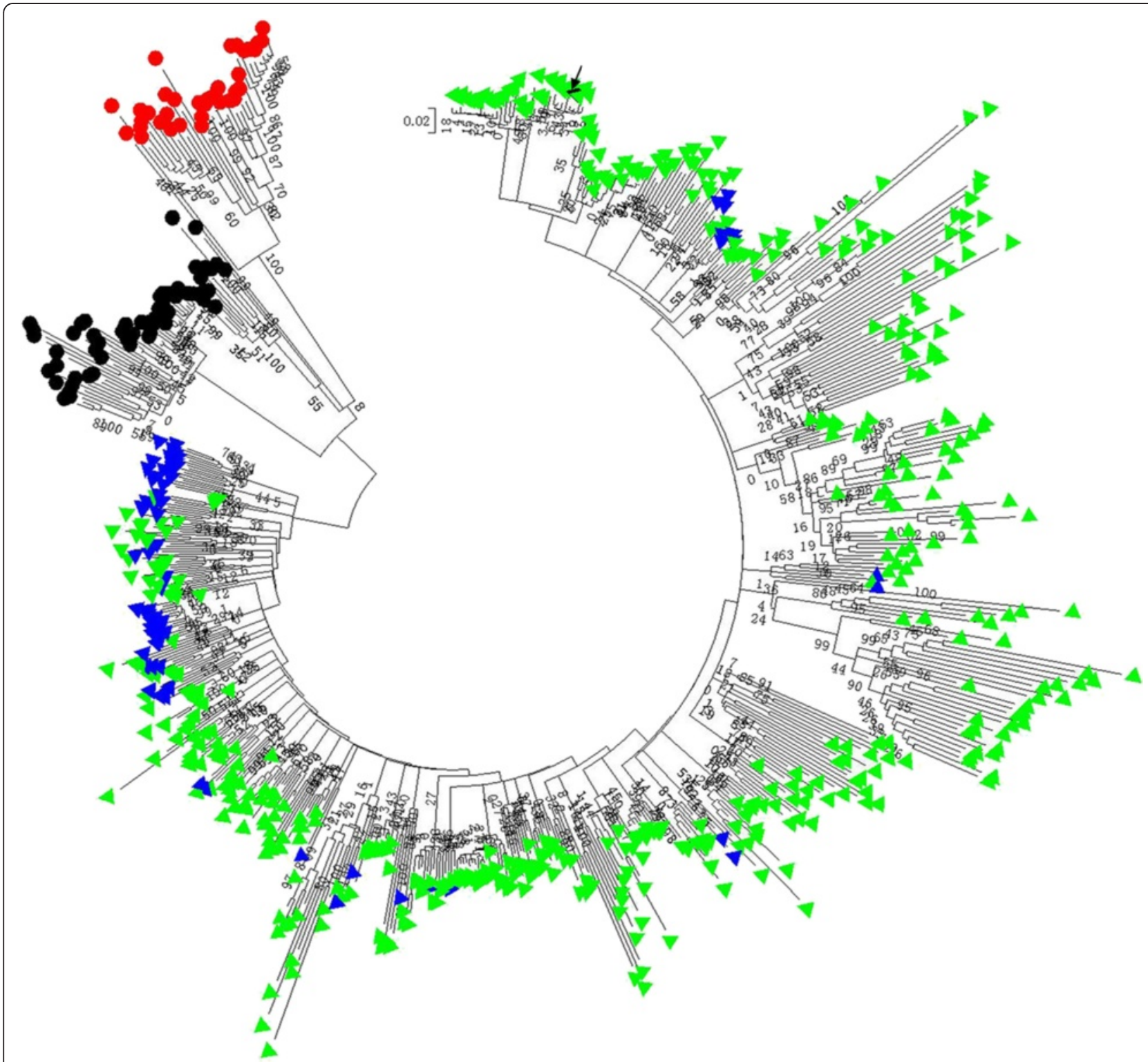

Fig. 4 Phylogenetic tree of complete Monkey King sequences from Brassicaceae genomes. Red and black circles indicate A. thaliana and A. Iyrata Monkey King sequences, respectively; Green and blue triangles indicate B. rapa and B. oleracea Monkey King sequences, respectively; the arrow points the Monkey King sequence in the BnFLC.A10 promoter in B. napus cultivar Tapidor

from the common ancestor and before B. napus speciation. In addition, inter-species polymorphisms caused by Monkey King insertions were also observed, e.g. the member SQ045005824 from B. rapa and the member C01-6 from B. oleracea (Fig. 5b and c).

\section{Monkey King DNA sequence was targeted for methylation and bound by nuclear proteins}

The Monkey King element identified in the promoter of BnFLC.A10 in our previous study [31], was used to check the potential ability of Monkey King to regulate gene expression via DNA methylation and was subjected to electrophoretic mobility shift assay (EMSA) analysis to check for interacting proteins. The methylation level of cytosine residues inside and flanking the Monkey King sequences was investigated using bisulfite sequencing. In B. napus cultivar Tapidor, cytosine methylation occurred in the Monkey King sequence, while no apparent cytosine methylation was observed in the flanking sequences; in cultivar Ningyou7, no DNA methylation occurred in the corresponding flanking regions (Fig. 6a). This means that DNA methylation was confined strictly to the Monkey King sequence.

The EMSA results clearly revealed that nuclear protein(s) extracted from Tapidor leaves specifically bound to a fragment (ES7) from the middle of the Monkey King sequence, almost entirely composed of A/T bases (Fig. $6 \mathrm{~b}$ 
Table 3 Summary of the insertion positions of complete Monkey King elements in the genomes of B. rapa and $A$. thaliana

\begin{tabular}{|c|c|c|c|c|}
\hline \multirow[t]{2}{*}{ Insertion position } & \multicolumn{2}{|l|}{ B. rapa } & \multicolumn{2}{|c|}{ A. thaliana } \\
\hline & $\begin{array}{l}\text { No. of } \\
\text { elements }\end{array}$ & $\begin{array}{l}\text { Percentage } \\
\text { of elements }\end{array}$ & $\begin{array}{l}\text { No. of } \\
\text { elements }\end{array}$ & $\begin{array}{l}\text { Percentage } \\
\text { of elements }\end{array}$ \\
\hline Gene & 24 & 4.8 & 2 & 5.3 \\
\hline 5'-flank(<1 kb) & 110 & 21.8 & 18 & 47.4 \\
\hline $5^{\prime}$-flank (1 kb to <2 kb) & 66 & 13.1 & 2 & 5.3 \\
\hline $5^{\prime}$-flank (2kb to $\left.<3 \mathrm{~kb}\right)$ & 33 & 6.5 & 1 & 2.6 \\
\hline $3^{\prime}$-flank(<1 kb) & 70 & 13.9 & 8 & 21.1 \\
\hline 3'-flank(1 kb to <2 kb) & 52 & 10.3 & 4 & 10.5 \\
\hline 3'-flank(2kb to <3 kb) & 20 & 4.0 & 0 & 0.0 \\
\hline $\begin{array}{l}\text { intergenic } \\
\text { Region (>3 kb) }\end{array}$ & 129 & 25.6 & 3 & 7.9 \\
\hline Total & 504 & 100.0 & 38 & 100.0 \\
\hline
\end{tabular}

and Additional file 4). Some Monkey King fragments (ES6, ES11, and ES12) were also recognized by nuclear proteins from Tapidor leaves; however, the binding was much weaker than to the ES7 fragment. Some fragments (e.g. ES4 and ES5) were non-specifically bound by nuclear proteins, because retarded bands were observed in both the noncompetitive (without plus unlabeled probes) and competitive (plus 50-fold unlabeled probes) binding assays. This result indicated that the Monkey King insertion produced new binding motifs for some nuclear proteins (probably transcriptional factors), which may regulate $B n F L C . A 10$ expression in winter varieties of B. napus.

\section{Detection of a Monkey King-related miRNA}

The B. napus Monkey King sequence was used to scan the microRNA database (miRBase) [32] and a miRNA known as bna-miR6031 was found to perfectly match to the internal region (499-522 bases) of the Monkey King sequence (Fig. 7a). The miRNA is 24 bp long and was firstly discovered as a new class in B. napus by Zhao et al. [33]. Further sequence analysis showed that the internal Monkey King

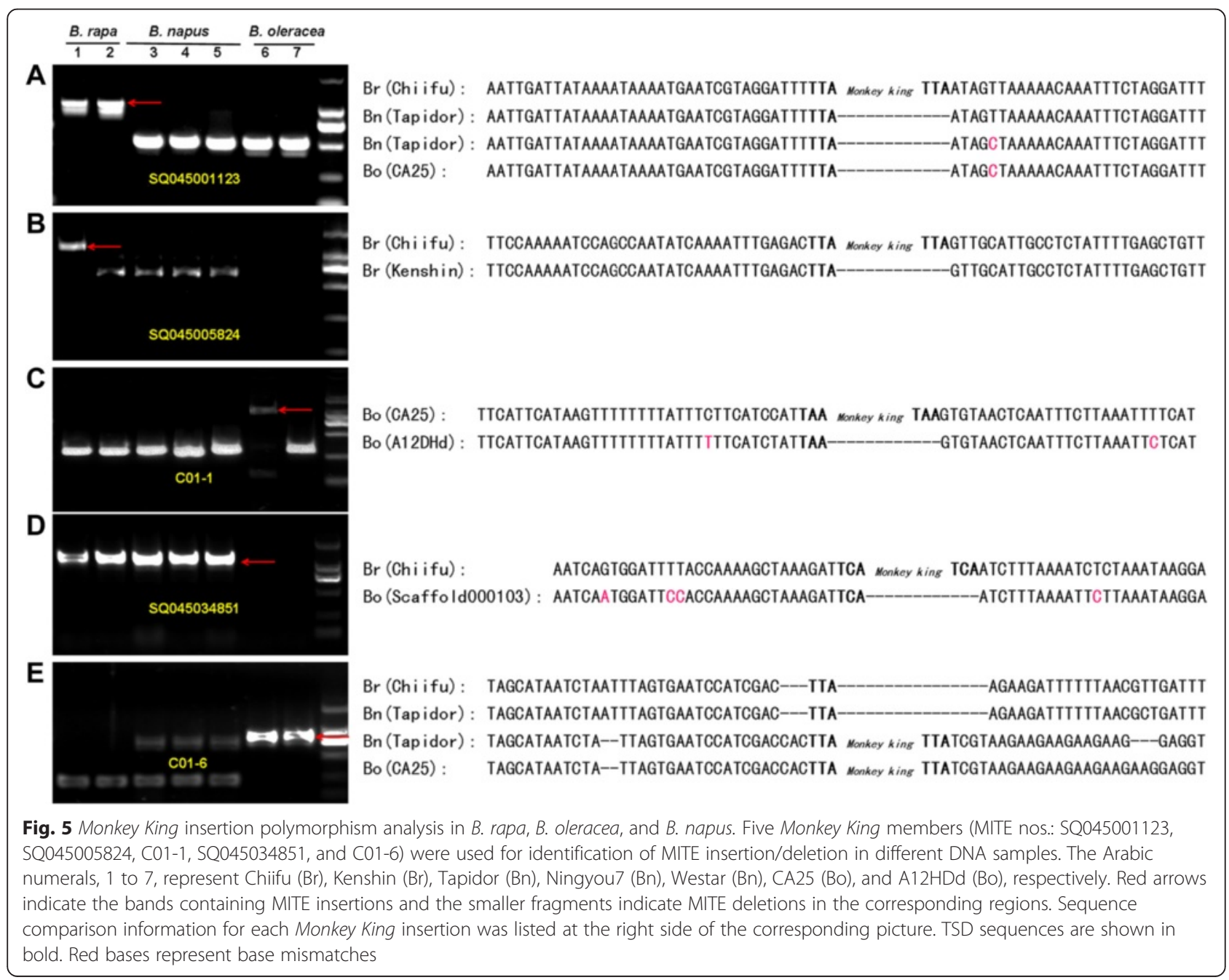


A
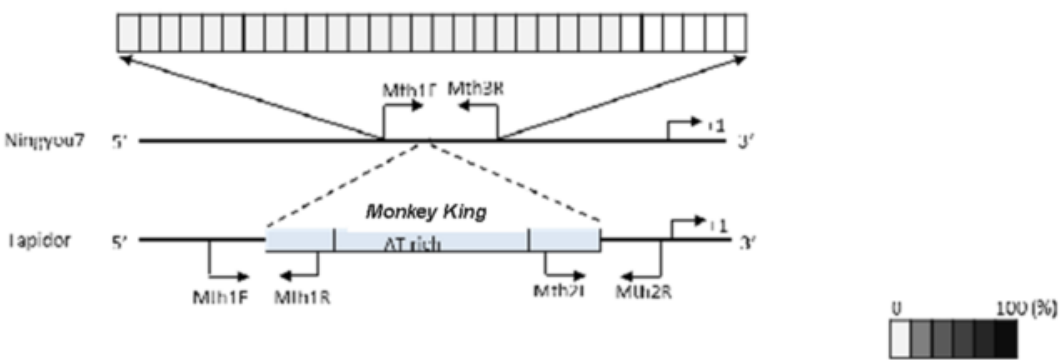

B
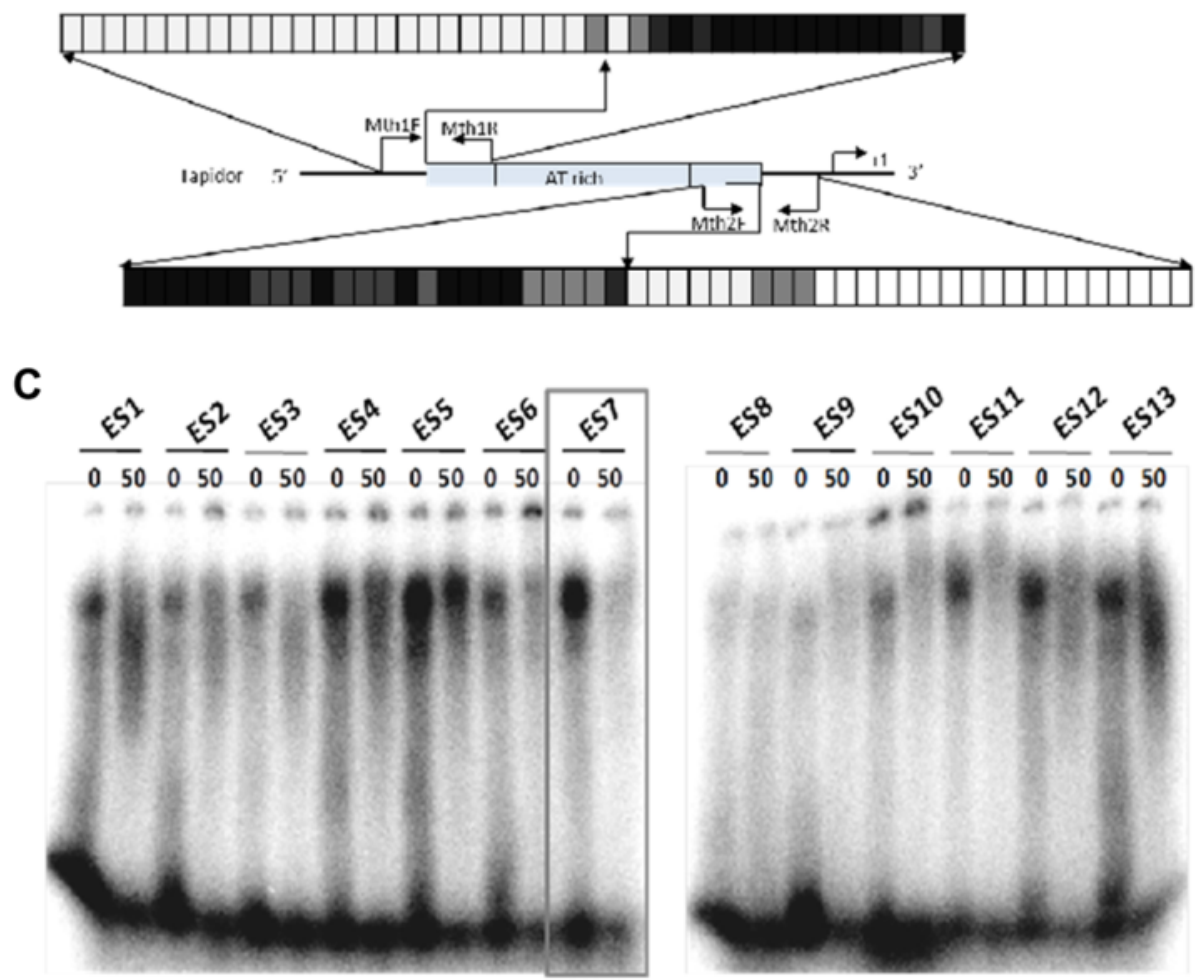

Fig. 6 DNA methylation and EMSA analysis of B. napus Monkey King. (a) and (b) DNA methylation detection results in the Monkey King sequence and its flanking sequences in the BnFLC.A10 promoter in Tapidor and Ningyou7. Each cell represents a cytosine. Blank cells denote no methylation. The higher the saturation is, the higher the DNA methylation level. (c) EMSA results in Tapidor. Thirteen probes (ES1-ES13) were derived from the Monkey King sequence. The binding ability of the probes to nuclear proteins from Tapidor leaves was analyzed by gel shift assays. Plus 50-fold (50) and without plus unlabeled probes (0) were used for the binding assays

sequence (499-586 bases) could form a stem-loop structure by itself (Fig. 7b), which suggested that bna-miR6031 is generated from Monkey King. In addition, BLAST analysis revealed that bna-miR6031 aligned well to several $B$. napus ESTs (Fig. 7c). This miRNA was used to search for similar miRNAs in the miRBase; however, none were found in other species, which suggested that bna-miR6031 is a Brassica species-specific miRNA.

\section{The Monkey King element decreases the activity of 35S} promoter in A. thaliana

To further investigate the effect of the Monkey King element on gene expression, we studied the influence of the Monkey King element on the activity of the 35S promoter that drives a GUS reporter gene. Two expression vectors, pBI121 (without Monkey King) and $\mathrm{pBI}^{\mathrm{m}} \mathrm{m}^{\mathrm{m}}$ (with Monkey King) (Fig. 8a) were used to produce transgenic A. thaliana via Agrobacterium-mediated floral dip transformation. Transcription of the GUS gene was assayed in transgene homozygous $\mathrm{T}_{3} A$. thaliana seedlings using quantitative real-time reverse transcription PCR (qRT-PCR). Seedlings hosting the $\mathrm{pBI} 121^{\mathrm{m}}$ construct had lower levels of the GUS transcript compared with the pBI121 seedlings (Fig. 8b). Chemical staining also showed that the $\mathrm{pBI} 121^{\mathrm{m}}$ seedlings displayed weaker GUS activities than the pBI121 seedlings (Fig. 8c). These 


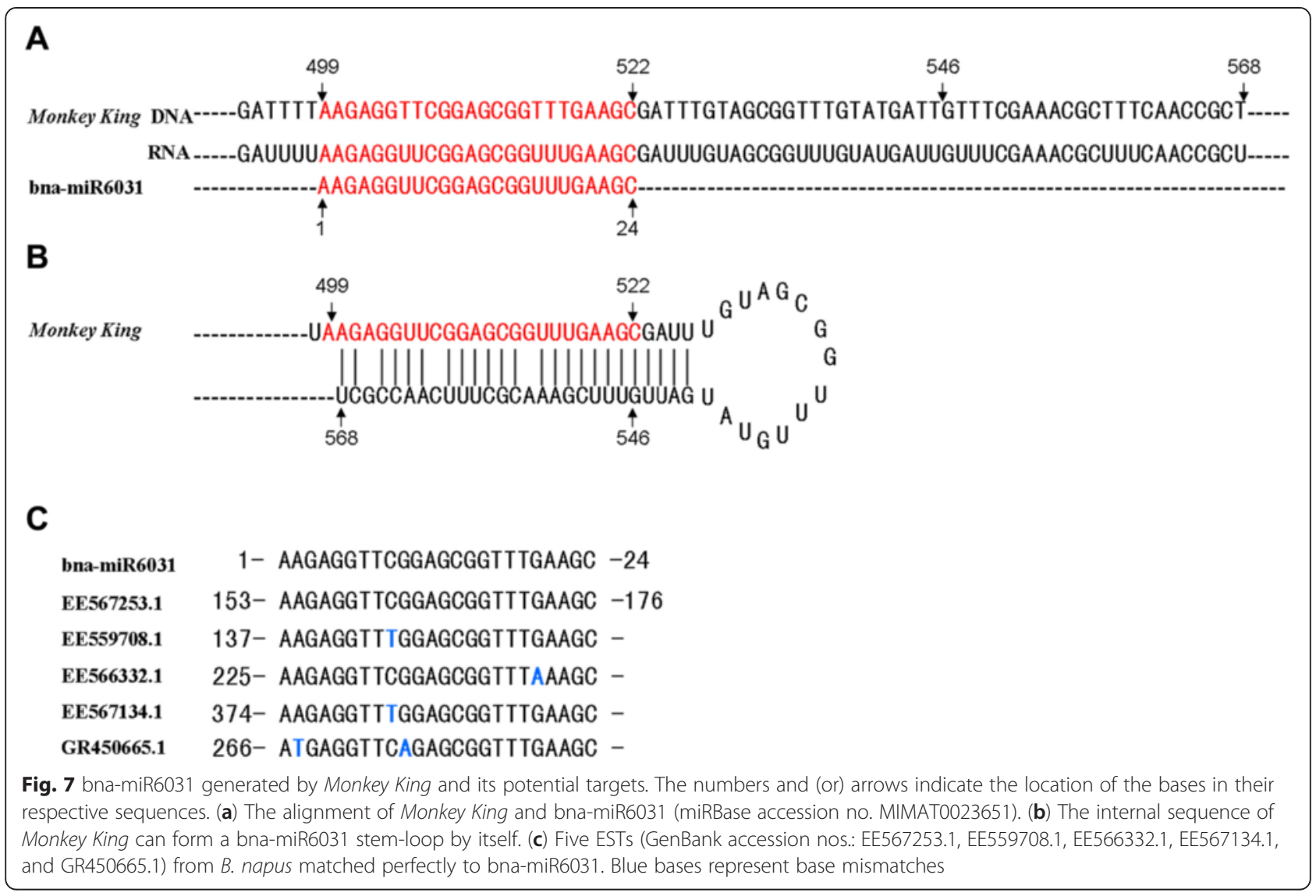

A

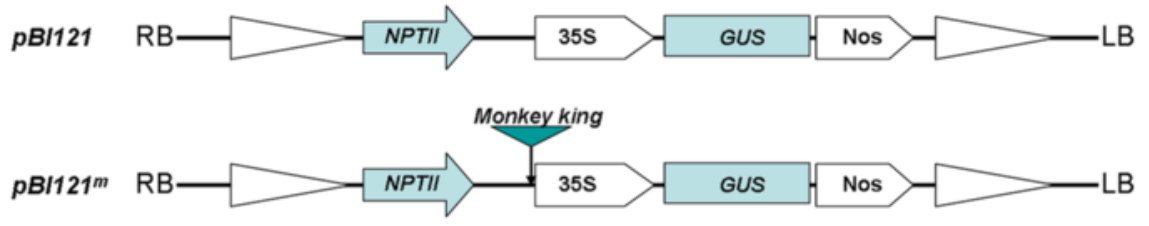

B

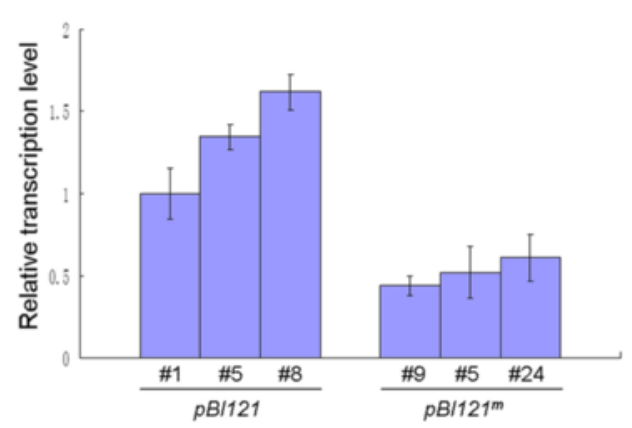

C

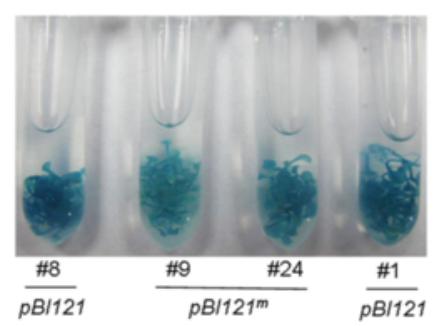

Fig. 8 The effect of the Monkey King element on the activity of the 35 S promoter in transgenic A. thaliana. (a) Schematic diagram of the pBI121 and $\mathrm{pBI} 121^{\mathrm{m}}$ constructs. The Monkey King sequence was inserted upstream of the $35 \mathrm{~S}$ promoter in the $\mathrm{pBI} 121^{\mathrm{m}}$ construct. (b) The relative transcriptional levels of the GUS gene in ten-day-old transgenic A. thaliana seedlings carrying the $\mathrm{pB} \mid 121$ and $\mathrm{pB} / 121^{\mathrm{m}}$ constructs, respectively. Error bars represent the standard deviation $(n=3)$. (c) Chemical staining for the GUS activity in ten-day-old transgenic $A$. thaliana seedlings 
results demonstrated that the Monkey King element decreased the activity of the $35 \mathrm{~S}$ promoter when inserted upstream of the promoter.

\section{Discussion}

In this study, we conducted molecular and genomic characterization of a Brassicaceae Tourist-like MITE, termed Monkey King. Monkey King possesses all of the typical features of Tourist MITEs and has the consensus TIR GGGC(orT)CTGACTGGTT. Interestingly, a similar TIR, GGGGNTGTTTGGTT, is present in kiddo and Tourist- $\mathrm{D}$ in rice and $\mathrm{Hbr}$ in maize [3, 34, 35]. However, no detectable similarity was observed with the internal sequences of these MITEs. The presence of this TIR in monocot and eudicot MITEs suggested that these MITEs may have evolved from a common ancestor, or that the sequences bearing this TIR easily create new MITE families with dissimilar internal sequences. Transposition of these MITEs may be mediated by the same transposase, because transposases recognize TIRs to ensure MITE mobility across the genome [36]. TIRs of the same family have been used to identify members of a MITE family [19, 37]. TIRs from one species could also be used to find novel MITE families in other species, because different MITE families from different species sometimes contain similar TIRs, such as Monkey King from the Brassicaceae family and kiddo, Tourist-D, and $\mathrm{Hbr}$ from the grass family.

Comparative analysis of Brassica species with A. thaliana revealed that, besides genome triplication and allopolyploidization, TEs contribute to the increased genome size of the Brassica species [38, 39]. In this study, MITE density analysis showed that Monkey King density in the $B$. rapa genome was three and 10 times more than that in the A. lyrata and A. thaliana genomes, respectively, demonstrating that the elements were actively amplified in the Brassica after divergence from Arabidopsis. On average, the B. rapa genome has the shortest complete Monkey King sequence, followed by the $A$. lyrata genome, while the $A$. thaliana genome has the longest sequence. Given the MITE density differences among these Brassicaceae genomes, we inferred that shorter Monkey King sequences may be more easily amplified than longer Monkey King sequences.

The high conservation of complete Monkey King sequences also suggested that they have amplified relatively recently in the Brassicaceae family. The high conservation of TIRs was also considered a sign of recent proliferation in the maize Tourist MITE ZmV1 [7] and the B. rapa Stowaway MITE BraSto [30]. Some Monkey King members have exactly the same TIR sequence, which supports the recent activity and ongoing mobilization of Monkey King. This hypothesis is also supported by the phylogenetic relationships among complete Monkey King sequences, because some species-specific clusters were found in the phylogenetic tree. In addition, the PCR amplification results from different Brassica DNA samples also suggested that some Monkey King insertions have arisen from independent activation in each species after speciation. In fact, our previous study suggested that the Monkey King insertion in the BnFLC.A10 promoter occurred in winter rapeseed after $B$. napus speciation [31]. These results indicated that some species-specific Monkey King members inserted into their respective genomes independently after allopolyploidization $\sim 7500$ years ago.

Monkey King is a high copy number Tourist MITE found in $B$. rapa, which parallels BRAMI-1, a recently identified high copy Stowaway MITE [40]. By contrast, In A. thaliana, we identified only 52 Monkey King members. The physical association between MITEs and genes showed that, in the $B$. rapa and $A$. thaliana genomes, most complete Monkey King elements were located in gene-rich regions, less than $3 \mathrm{~kb}$ from genes. Many members were within less than $1 \mathrm{~kb}$ from a gene, while a few members were within introns. Although the MITE density and copy number showed obvious differences between $B$. rapa and $A$. thaliana genomes, a similar distribution trend was observed in the two genomes: the Monkey King sequences were most abundant in the vicinity of genes. The insertion preference of this Brassicaceae Tourist MITE is similar to that of the two Brassica Stowaway MITEs, BroSto and BRAMI-1 [30, 40]. MITEs inserted in gene regulatory regions can modify gene transcriptional activity and change gene expression levels $[13,41]$. In $A$. thaliana, nearly half of the members were within less than $0.5 \mathrm{~kb}$ from a UTR and two members fell within UTR regions. Additionally, 30 of 67 Brassica ESTs carrying Monkey King fragments matched the annotated $B$. rapa and (or) A. thaliana genes. Thus, Monkey King insertions could play a role in gene regulation and evolution in Brassicaceae.

Although many MITEs have been found in plant genomes and are associated with genes, few studies have examined the effects of MITE insertions on neighboring gene expression [18, 20, 22]. In Solanaceae, some MITEs generate small RNAs, thus playing a direct role in gene regulation through the small RNA silencing pathway [22]. In rice, sequences within mPing were considered as enhancers that render adjacent genes stress inducible [18]. Promoter activity analysis revealed that the MITE kiddo was responsible for up to $20 \%$ of neighboring gene expression in both transient and stably transformed rice calli [20]. Moreover, when kiddo DNA methylation was blocked with 5-azaC, ubiquitin2 transcript accumulation increased threefold [20]. This indicated kiddo has a dual function in regulating gene expression. In our previous study, the Monkey King insertion upstream of 
BnFLC.A10 was positively associated with induced BnFLC.A10 expression during vernalization [31]. In this study, Monkey King DNA methylation in the BnFLC.A10 promoter was observed. This suggested that epigenetic modification may regulate BnFLC.A10 expression by changing the Monkey King DNA methylation status. Monkey King contains important motifs, such as TATA-box and CAAT-box [31]. Certain transcription factors probably bind to the motifs to change neighboring gene expression profiles. Indeed, a fragment (ES7) containing putative TATA-box motifs within Monkey King bound nuclear protein(s) extracted from Tapidor leaves. These results suggest that Monkey King insertions can produce new regulatory sites, which probably recruit new transcription factors. New alleles caused by MITE insertions may benefit the host by producing useful allelic variants and novel regulatory mechanisms. In fact, BnFLC.A10 allelic diversity caused by the Monkey King insertion is one of the major causes of differentiation of winter and spring B. napus genotypes [31]. In addition, identification of the Monkey King-related miRNA, bna-miR6031, and its potential targets, indicated that the Monkey King silencing pathway may be involved in expression regulation of genes bearing Monkey King insertions. In Arabidopsis, a transposable element was inserted in an intron of $F L C$, resulting in low FLC RNA levels. The intronic TE renders FLC subject to repressive chromatin modifications mediated by TE-derived siRNAs [42]. In wheat, a MITE (MITE_VRN) inserted in the promoter of VRN-A1a and influenced the flowering [43, 44]. Recent report showed that the MITE_VRN also possessed sequences of a miRNA (TamiR1123) [45]. Whether or not bna-miR6031 regulates $B n F L C . A 10$ expression through chromatin modifications of the Monkey King sequence in the BnFLC.A10 promoter remains to be investigated. bna-miR6301 is a new class of miRNA found in B. napus [33] and may be specific to Brassica species because it may be generated by a certain Monkey King sequence. Moreover, no similar miRNAs from other species are present in miRbase. To date, many MITEs have only been identified in a given species, which suggests that MITEs can generate speciesspecific small RNAs. The Monkey King insertion was positively associated with BnFLC.A10 expression [31], however, our transgenic $A$. thaliana experiment revealed that the Monkey King element inhibited promoter activity when inserted upstream region of a promoter. These results indicate that a new Monkey King insertion may enhance or repress the expression of a gene with which it is associated, by causing DNA epigenetic modification, nuclear protein binding sites and(or) possible miRNA-mediated regulation. Given the wide distribution of Monkey King elements in some Brassicaceae genomes, this Tourist MITE family may contribute considerable phenotypic diversity to Brassicaceae plants.

\section{Conclusions}

In this study, we characterized a Brassicaceae Touristlike MITE family Monkey King. Comparative analysis of Brassica species with Arabidopsis species revealed its putative role in the evolution of Brassicaceae genomes. Phylogenetic analysis and investigation of intra- and inter-species polymorphisms supported recent proliferation of Monkey King in Brassica species. Monkey King elements are closely associated with genes and influence gene regulation and evolution. DNA methylation detection, EMSA analysis, identification of a Monkey King-related miRNA, and transgenic experiments suggested that Monkey King insertions could regulate gene expression and genome evolution in the Brassicaceae family in a variety of ways, such as epigenetic modification and new regulatory motif production.

\section{Methods}

Mining and characterization of Monkey King sequences in Brassicaceae genomes

The Monkey King sequence inserted in the BnFLC.A10 promoter was first used as a query to search for similar sequences in the NCBI database and was employed to find its coding capacity. When similar sequences were only found in Brassicaceae genomes, the BLAST program was used to screen the homologous sequences in the published plant MITE databases (P-MITE) [11]. All Monkey King sequences in B. rapa and A. lyrata genomes were downloaded from the P-MITE database for further analysis. Monkey King homologous sequences were also identified in A. thaliana genome in the NCBI database by BLAST screening (an expected value $<1 \mathrm{e}^{-10}$ ) and manual inspection. Additionally, complete Monkey King sequences (with TSDs at the two ends of one Monkey King sequence) were identified in the preliminary assembled B. oleracea genome sequence (http://brassicadb.org/). Nucleotide composition of the complete Monkey King sequences was computed in MEGA 5 [46]. Conservation of the TIRs of the complete Monkey King sequences among four Brassicaceae plant genomes, B. rapa, B. oleracea, A. thaliana and A. lyrata, was visualized using the program Pictograms (http://genes.mit.edu/pictogram.html). Then, the Monkey King sequence was used against the microRNA database (miRBase) (http://www.mirbase.org/ search.shtml) [32] to find miRNAs.

\section{Phylogenetic analysis of Monkey King}

All of the complete Monkey King sequences were used for multiple sequence alignment using ClustalW in MEGA 5 [46]. Phylogenetic trees were constructed with the neighbor-joining method in MEGA 5 [46] following manual refinement. Bootstraps with 1000 replicates were performed to assess node support using the p-distance model. 


\section{Characterization of Monkey King insertion sites}

The insertion sites of 504 and 38 complete Monkey King elements and their flanking regions were annotated using the B. rapa genome database (http://brassicadb.org/) and the $A$. thaliana genome database (http://www.arabi dopsis.org/), respectively. The distances between Monkey King elements and their respective nearest genes were calculated for analyzing the relationship between the elements and genes. Additionally, Potential transcription activity of Monkey King was examined by searching Brassica EST database at NCBI. The Brassica ESTs carrying Monkey King fragments were used for localizing the Monkey King fragments in their respective genes, by comparison with the corresponding annotated $B$. rapa genes (http://brassicadb.org/brad/blastPage.php) and A. thaliana genes (http://www.arabidopsis.org/wublast/ index2.jsp).

\section{Survey of intra- and inter-species genomic variations caused by Monkey King insertions in B. rapa, B. oleracea, and $B$. napus}

Intra- and inter-species genomic variations caused by Monkey King insertions were checked by PCR amplification. The flanking sequences of 5 Monkey King members were extracted for primer design (Additional file 5). PCR amplification was performed with DNA samples from two $B$. rapa (Chiifu and Kenshin), three $B$. napus (Tapidor, Ningyou7 and Westar) and two $B$. oleracea (CA25 and A12DHd) accessions. Sequencing and sequence comparisons were performed for further identification of the Monkey King insertions.

\section{DNA methylation detection}

Previous studies have identified the Monkey King member inserted in the $B n A 10 . F L C$ promoter in winter-type $B$. napus cultivar Tapidor, compared with that in a semiwinter-type $B$. napus cultivar Ningyou7 [31]. Therefore, the two cultivars were used to check the potential ability of Monkey King to regulate gene expression.

Genomic DNA was extracted from young Tapidor and Ningyou7 leaves, and $2 \mu \mathrm{g}$ DNA were subjected to bisulfite treatment using the EpiTect Bisulfite Kit (Qiagen) following the manufacturer's instructions. The Monkey King member in the BnFLC.A10 promoter was selected to detect the DNA methylation level of its inside and flanking sequences. The primers (Additional file 5) were designed using the software Kismeth (http://katahdin.mssm.edu/kismeth) [47]. Ten clones were sequenced for each amplified product for DNA methylation analysis. The positive control was set to assure that the efficiency of the bisulfite treatment and that all of the non-methylated cytosines were converted to uracil [48].

\section{Electrophoretic mobility shift assay (EMSA) analysis}

Leaves (6 g) were harvested from Tapidor seedlings. Protein extraction was performed as described by Mazabal et al. [49] with some modification. After grinding the leaves in liquid nitrogen, the powder was transferred into $40 \mathrm{ml}$ of homogenizing buffer (10 mM Hepes, $\mathrm{pH}$ 7.8, $10 \mathrm{mM} \mathrm{KCl}, 10 \mathrm{mM} \mathrm{MgCl}$, $5 \mathrm{mM}$ EDTA, $1 \mathrm{mM}$ DTT, $200 \mathrm{mM}$ PMSF, $250 \mathrm{mM}$ sucrose, and $0.5 \%$ Triton $\mathrm{X}-100$ ) and stirred for over $20 \mathrm{~min}$. The mixture was filtered with a Miracloth and the supernatant was centrifuged at $3000 \times \mathrm{g}$ for $20 \mathrm{~min}$ at $4{ }^{\circ} \mathrm{C}$. The pellet was resuspended in a low salt buffer (20 mM Hepes, $\mathrm{pH} 7.8$, $20 \mathrm{mM} \mathrm{KCl}, 1.5 \mathrm{mM} \mathrm{MgCl}_{2}, 25 \%$ glycerol, $200 \mathrm{mM}$ EDTA, $500 \mathrm{mM}$ DTT, and $200 \mathrm{mM}$ PMSF) and centrifuged at $3000 \times \mathrm{g}$ for $10 \mathrm{~min}$ at $4^{\circ} \mathrm{C}$ (this step was repeated for 2 times). The pellet was suspended in a high salt buffer (20 mM Hepes, pH 7.8, $1 \mathrm{M} \mathrm{KCl,} 1.5 \mathrm{mM}$ $\mathrm{MgCl}_{2}, 25 \%$ glycerol, $200 \mathrm{mM}$ EDTA, $500 \mathrm{mM}$ DTT, and $200 \mathrm{mM}$ PMSF) in a volume of the estimated PNV (pellet nuclear volume). The nuclear lysate was incubated for $30 \mathrm{~min}$ at $4{ }^{\circ} \mathrm{C}$ with shaking and then centrifuged at $12000 \times \mathrm{g}$ for $20 \mathrm{~min}$ at $4{ }^{\circ} \mathrm{C}$. The supernatant was dialyzed in the low salt buffer for over $1 \mathrm{~h}$. Finally, the protein content was measured by the Coomassie Brilliant Blue method. The EMSA was conducted as described by Hellman [50]. Probes that covered the Monkey King sequence were synthesized (Additional file 4).

\section{Vector construction, plant transformation, and GUS activity analysis}

The Monkey King sequence was amplified from a $B$. napus BAC (JBnB75D10) carrying the Monkey King insertion in the BnA10.FLC promoter [31] using specific primers (Additional file 5) and cloned into the HindIII site of the binary vector pBI121 (Clontech, USA). The correct orientation was confirmed by DNA sequencing. The original pBI121 vector was used as a control. The two vectors were introduced into Agrobacterium tumefaciens strain LBA4404 and then transformed into $A$. thaliana using the floral dip method [51]. A segregation assay was used to identify lines harboring a single transgene copy, by culturing the $\mathrm{T}_{2}$ transgenic plants on halfstrength MS medium containing $50 \mathrm{mg} \mathrm{L}^{-1}$ of kanamycin. $\mathrm{T}_{3}$ lines carrying a single transgene copy were assumed to be homozygous lines when they did not segregate for kanamycin resistance.

$\mathrm{T}_{3}$ homozygous lines were used to study the effect of the Monkey King element on gene expression. Ten-dayold seedlings grown on MS medium were harvested for RNA collection and GUS staining. Total RNA was extracted from about 25 seedlings using a Universal Plant Total RNA Extraction Kit (Cat.\# RP3301, BioTeke, Beijing, China). RNA samples were treated with DNaseI and used for reverse transcription using M-MLV reverse 
transcriptase (Promega). SYBR-Green qRT-PCR was used to quantify the transcription level of the GUS gene in transgenic seedlings. The $25 \mu \mathrm{L}$ reaction contained $12.5 \mu \mathrm{L}$ SYBR Green PCR Master Mix (Toyobo), 600 nM primers, and $2 \mu \mathrm{L}$ of $5 \times$ diluted cDNA sample. The PCR profile was as follows: $95{ }^{\circ} \mathrm{C}$ for $1 \mathrm{~min}$, followed by 40 cycles of $95^{\circ} \mathrm{C}$ for $5 \mathrm{sec}$ and $60^{\circ} \mathrm{C}$ for $1 \mathrm{~min}$. Fluorescence data were collected after the $60{ }^{\circ} \mathrm{C}$ step. Three replicate reactions were performed with each cDNA sample and individual primer pairs (Additional file 5). Chemical staining for GUS activity was performed as described by Jefferson et al. [52] with minor modifications. Seedlings were soaked in the GUS assay solution and incubated at $37^{\circ} \mathrm{C}$ for $24 \mathrm{~h}$. The GUS assay solution contained $1 \mathrm{~g} \mathrm{~L}^{-1} \mathrm{X}$-Gluc, $0.5 \mathrm{mM} \mathrm{K} \mathrm{K}_{3} \mathrm{Fe}(\mathrm{CN})_{6}, 0.5 \mathrm{mM}$ $\mathrm{K}_{4} \mathrm{Fe}(\mathrm{CN})_{6}, 10 \mathrm{mM} \mathrm{Na}{ }_{2} \mathrm{EDTA}, 0.1 \%$ (v/v) Triton X-100, $20 \%(\mathrm{v} / \mathrm{v})$ methanol, and $100 \mathrm{mM}$ sodium phosphate (pH 7.0). After staining, the samples were rinsed with ethanol and photographed.

\section{Availability of supporting data}

Phylogenetic data is available in the TreeBASE as accession number S17406 (http://purl.org/phylo/treebase/phylows/ study/TB2:S17406 ).

\section{Additional files}

\section{Additional file 1: Physical position and annotation of 504 complete} sequences of Monkey King members on the B. rapa genome.

Additional file 2: Physical position and annotation of 38 complete sequences of Monkey King members on the A. thaliana genome.

Additional file 3: Characterization of the significant similarities obtained from BLASTN searches using Monkey King as the query against Brassica EST database from NCBI.

Additional file 4: Sequences of probes used in EMSA.

Additional file 5: Sequence information for primers used for Monkey King insertion polymorphisms analysis, DNA methylation analysis, vector construction and GUS gene expression analysis.

\footnotetext{
Abbreviations

MITE: Miniature inverted repeat transposable element; EST: Expressed sequence tag; TIR: Terminal inverted repeats; TSD: Target site duplications; FLC: FLOWERING LOCUS C; EMSA: Electrophoretic mobility shift assay; UTR: Untranslated region; qRT-PCR: Quantitative real-time reverse transcription PCR.
}

\section{Competing interests}

The authors declare that they have no competing interests.

\section{Authors' contributions}

$\mathrm{SD}, \mathrm{JH}, \mathrm{YL}$, and JM designed the study, and wrote the manuscript. SD and JH performed the bioinformatics analysis. XJ extracted some sequences. SD, JH, $\mathrm{CL}$, and QX carried out the molecular experiment. SD performed the transgenic experiment. JW, XZ, and JZ provided suggestions for data analysis, paper writing and revised the manuscript. JM, $\mathrm{YL}$, and $\mathrm{JZ}$ supported the study. All authors read and approved the final manuscript.

\section{Acknowledgements}

The authors gratefully acknowledge the financial support provided by the National Natural Science Foundation of China (31100876, 31371657), and the National Key Technology Support Program (2012BAD49G00).

\section{Author details}

${ }^{1}$ National Key Lab of Crop Genetic Improvement, Huazhong Agricultural University, Wuhan, Hubei 430070, China. ${ }^{2}$ Crop Designing Centre, Henan Academy of Agricultural Sciences, Zhenzhou, Henan 450002, China.

Received: 10 February 2015 Accepted: 7 April 2015

Published online: 19 June 2015

\section{References}

1. Bureau TE, Ronald PC, Wessler SR. A computer-based systematic survey reveals the predominance of small inverted-repeat elements in wild-type rice genes. Proc Natl Acad Sci U S A. 1996;93(16):8524-9.

2. Bureau TE, Wessler SR. Tourist: a large family of small inverted repeat elements frequently associated with maize genes. Plant Cell. 1992;4(10):1283-94.

3. Bureau TE, Wessler SR. Mobile inverted-repeat elements of the Tourist family are associated with the genes of many cereal grasses. Proc Natl Acad Sci U S A. 1994;91(4):1411-5.

4. Bureau TE, Wessler SR. Stowaway: a new family of inverted repeat elements associated with the genes of both monocotyledonous and dicotyledonous plants. Plant Cell. 1994;6(6):907-16.

5. Zhang X, Jiang N, Feschotte C, Wessler SR. PIF- and Pong-like transposable elements: distribution, evolution and relationship with Tourist-like miniature inverted-repeat transposable elements. Genetics. 2004;166(2):971-86.

6. Feschotte $C$, Mouches $C$. Evidence that a family of miniature inverted-repeat transposable elements (MITEs) from the Arabidopsis thaliana genome has arisen from a pogo-like DNA transposon. Mol Biol Evol. 2000;17(5):730-7.

7. Zerjal T, Joets J, Alix K, Grandbastien MA, Tenaillon MI. Contrasting evolutionary patterns and target specificities among three Tourist-like MITE families in the maize genome. Plant Mol Biol. 2009;71(1-2):99-114.

8. Depra M, Ludwig A, Valente VL, Loreto EL. Mar, a MITE family of hAT transposons in Drosophila. Mob DNA. 2012;3(1):13.

9. Moreno-Vazquez S, Ning J, Meyers BC. hATpin, a family of MITE-like hAT mobile elements conserved in diverse plant species that forms highly stable secondary structures. Plant Mol Biol. 2005;58(6):869-86.

10. Yang G, Hall TC. MDM-1 and MDM-2: two mutator-derived MITE families in rice. J Mol Evol. 2003;56(3):255-64

11. Chen J, Hu Q, Zhang Y, Lu C, Kuang H. P-MITE: a database for plant miniature inverted-repeat transposable elements. Nucleic Acids Res. 2013;42(Database issue):D1176-81.

12. Han MJ, Shen YH, Gao YH, Chen LY, Xiang ZH, Zhang Z. Burst expansion, distribution and diversification of MITEs in the silkworm genome. BMC Genomics. 2010;11:520.

13. Lu C, Chen J, Zhang Y, Hu Q, Su W, Kuang H. Miniature inverted-repeat transposable elements (MITEs) have been accumulated through amplification bursts and play important roles in gene expression and species diversity in Oryza sativa. Mol Biol Evol. 2012;29(3):1005-17.

14. Jiang $\mathrm{N}$, Bao Z, Zhang $X$, Hirochika $\mathrm{H}$, Eddy SR, McCouch SR, et al. An active DNA transposon family in rice. Nature. 2003;421(6919):163-7.

15. Kikuchi K, Terauchi K, Wada M, Hirano HY. The plant MITE mPing is mobilized in anther culture. Nature. 2003;421(6919):167-70.

16. Nakazaki T, Okumoto Y, Horibata A, Yamahira S, Teraishi M, Nishida H, et al. Mobilization of a transposon in the rice genome. Nature. 2003:421(6919):170-2.

17. Naito K, Cho E, Yang G, Campbell MA, Yano K, Okumoto Y, et al. Dramatic amplification of a rice transposable element during recent domestication. Proc Natl Acad Sci U S A. 2006;103(47):17620-5.

18. Naito K, Zhang F, Tsukiyama T, Saito H, Hancock CN, Richardson AO, et al. Unexpected consequences of a sudden and massive transposon amplification on rice gene expression. Nature. 2009;461(7267):1130-4.

19. Santiago N, Herraiz C, Goni JR, Messeguer X, Casacuberta JM. Genome-wide analysis of the Emigrant family of MITEs of Arabidopsis thaliana. Mol Biol Evol. 2002;19(12):2285-93.

20. Yang G, Lee YH, Jiang Y, Shi X, Kertbundit S, Hall TC. A two-edged role for the transposable element Kiddo in the rice ubiquitin2 promoter. Plant Cell. 2005;17(5):1559-68. 
21. Henderson IR, Jacobsen SE. Epigenetic inheritance in plants. Nature. 2007:447(7143):418-24.

22. Kuang H, Padmanabhan C, Li F, Kamei A, Bhaskar PB, Ouyang S, et al. Identification of miniature inverted-repeat transposable elements (MITEs) and biogenesis of their siRNAs in the Solanaceae: new functional implications for MITEs. Genome Res. 2009;19(1):42-56.

23. Chalhoub B, Denoeud F, Liu S, Parkin IA, Tang H, Wang X, et al. Plant genetics. Early allopolyploid evolution in the post-Neolithic Brassica napus oilseed genome. Science. 2014;345(6199):950-3.

24. Rana D, van den Boogaart T, O'Neill CM, Hynes L, Bent E, Macpherson L et al. Conservation of the microstructure of genome segments in Brassica napus and its diploid relatives. Plant J. 2004;40(5):725-33.

25. Liu S, Liu Y, Yang X, Tong C, Edwards D, Parkin IA, et al. The Brassica oleracea genome reveals the asymmetrical evolution of polyploid genomes. Nat Commun. 2014;5:3930.

26. Qian W, Chen X, Fu D, Zou J, Meng J. Intersubgenomic heterosis in seed yield potential observed in a new type of Brassica napus introgressed with partial Brassica rapa genome. Theor Appl Genet. 2005;110(7):1187-94.

27. Li M, Qian W, Meng J, Li Z. Construction of novel Brassica napus genotypes through chromosomal substitution and elimination using interploid species hybridization. Chromosome Res. 2004;12(5):417-26.

28. Cho K, O'Neill CM, Kwon SJ, Yang TJ, Smooker AM, Fraser F, et al. Sequencelevel comparative analysis of the Brassica napus genome around two stearoyl-ACP desaturase loci. Plant J. 2010;61(4):591-9.

29. Cheung F, Trick M, Drou N, Lim YP, Park JY, Kwon SJ, et al. Comparative analysis between homoeologous genome segments of Brassica napus and its progenitor species reveals extensive sequence-level divergence. Plant Cell. 2009;21(7):1912-28.

30. Sarilar V, Marmagne A, Brabant $P$, Joets J, Alix K. BraSto, a Stowaway MITE from Brassica: recently active copies preferentially accumulate in the gene space. Plant Mol Biol. 2011;77(1-2):59-75

31. Hou J, Long Y, Raman H, Zou X, Wang J, Dai S, et al. A Tourist-like MITE insertion in the upstream region of the BnFLC.A10 gene is associated with vernalization requirement in rapeseed (Brassica napus L.). BMC Plant Biol. 2012;12:238

32. Kozomara A, Griffiths Jones S. miRBase: integrating microRNA annotation and deep-sequencing data. Nucleic Acids Res. 2010;39(Database issue):D152-7.

33. Zhao YT, Wang M, Fu SX, Yang WC, Qi CK, Wang XJ. Small RNA profiling in two Brassica napus cultivars identifies microRNAs with oil production- and development-correlated expression and new small RNA classes. Plant Physiol. 2012;158(2):813-23.

34. Yang G, Dong J, Chandrasekharan MB, Hall TC. Kiddo, a new transposable element family closely associated with rice genes. Mol Genet Genomics. 2001;266(3):417-24.

35. Zhang Q, Arbuckle J, Wessler SR. Recent, extensive, and preferential insertion of members of the miniature inverted-repeat transposable element family Heartbreaker into genic regions of maize. Proc Natl Acad Sci U S A. 2000;97(3):1160-5.

36. Feschotte C, Osterlund MT, Peeler R, Wessler SR. DNA-binding specificity of rice mariner-like transposases and interactions with Stowaway MITEs. Nucleic Acids Res. 2005;33(7):2153-65.

37. Lyons M, Cardle L, Rostoks N, Waugh R, Flavell AJ. Isolation, analysis and marker utility of novel miniature inverted repeat transposable elements from the barley genome. Mol Genet Genomics. 2008;280(4):275-85.

38. Zhang X, Wessler SR. Genome-wide comparative analysis of the transposable elements in the related species Arabidopsis thaliana and Brassica oleracea. Proc Natl Acad Sci U S A. 2004;101(15):5589-94.

39. Wang $X$, Wang H, Wang J, Sun R, Wu J, Liu S, et al. The genome of the mesopolyploid crop species Brassica rapa. Nat Genet. 2011:43(10):1035-9.

40. Sampath P, Lee SC, Lee J, Izzah NK, Choi BS, Jin M, et al. Characterization of a new high copy Stowaway family MITE, BRAMI-1 in Brassica genome. BMC Plant Biol. 2013;13:56.

41. Casacuberta JM, Santiago N. Plant LTR-retrotransposons and MITEs: control of transposition and impact on the evolution of plant genes and genomes. Gene. 2003;311:1-11.

42. Liu J, He Y, Amasino R, Chen X. siRNAs targeting an intronic transposon in the regulation of natural flowering behavior in Arabidopsis. Genes Dev. 2004;18(23):2873-8.
43. Yan L, Helguera M, Kato K, Fukuyama S, Sherman J, Dubcovsky J. Allelic variation at the VRN-1 promoter region in polyploid wheat. Theor Appl Genet. 2004;109(8):1677-86.

44. Yan L, Loukoianov A, Tranquilli G, Helguera M, Fahima T, Dubcovsky J. Positional cloning of the wheat vernalization gene VRN1. Proc Natl Acad Sci U S A. 2003;100(10):6263-8.

45. Yu M, Carver BF, Yan L. TamiR1123 originated from a family of miniature inverted-repeat transposable elements (MITE) including one inserted in the Vrn-A1a promoter in wheat. Plant Sci. 2014;215-216:117-23.

46. Tamura K, Peterson D, Peterson N, Stecher G, Nei M, Kumar S. MEGA5: molecular evolutionary genetics analysis using maximum likelihood, evolutionary distance, and maximum parsimony methods. Mol Biol Evol. 2011;28(10):2731-9.

47. Gruntman E, Qi Y, Slotkin RK, Roeder T, Martienssen RA, Sachidanandam R. Kismeth: analyzer of plant methylation states through bisulfite sequencing BMC Bioinformatics. 2008;9:371.

48. Wang J, Wang C, Long Y, Hopkins C, Kurup S, Liu K, et al. Universal endogenous gene controls for bisulphite conversion in analysis of plant DNA methylation. Plant Methods. 2011;7:39.

49. Marzabal P, Busk PK, Ludevid MD, Torrent M. The bifactorial endosperm box of gamma-zein gene: characterisation and function of the Pb3 and GZM cis-acting elements. Plant J. 1998;16(1):41-52

50. Hellman LM, Fried MG. Electrophoretic mobility shift assay (EMSA) for detecting protein-nucleic acid interactions. Nat Protoc. 2007;2(8):1849-61.

51. Clough SJ, Bent AF. Floral dip: a simplified method for Agrobacteriummediated transformation of Arabidopsis thaliana. Plant J. 1998;16(6):735-43.

52. Jefferson RA, Kavanagh TA, Bevan MW. GUS fusions: beta-glucuronidase as a sensitive and versatile gene fusion marker in higher plants. EMBO J. 1987;6(13):3901-7.

\section{Submit your next manuscript to BioMed Central and take full advantage of:}

- Convenient online submission

- Thorough peer review

- No space constraints or color figure charges

- Immediate publication on acceptance

- Inclusion in PubMed, CAS, Scopus and Google Scholar

- Research which is freely available for redistribution 